\title{
The reaction of ethylene with hydroxyl radicals: a theoretical study
}

\author{
Juan P. Senosiain ${ }^{*}$, Stephen J. Klippenstein ${ }^{\mathrm{b}}$, James A. Miller ${ }^{* a}$ \\ (a) Combustion Research Facility, Sandia National Laboratories, Livermore, CA 94551-0969 MS 9055, USA \\ (b) Argonne National Laboratory, 9700 S. Cass Avenue,Argonne, IL 60439
}

Supplementary Materials

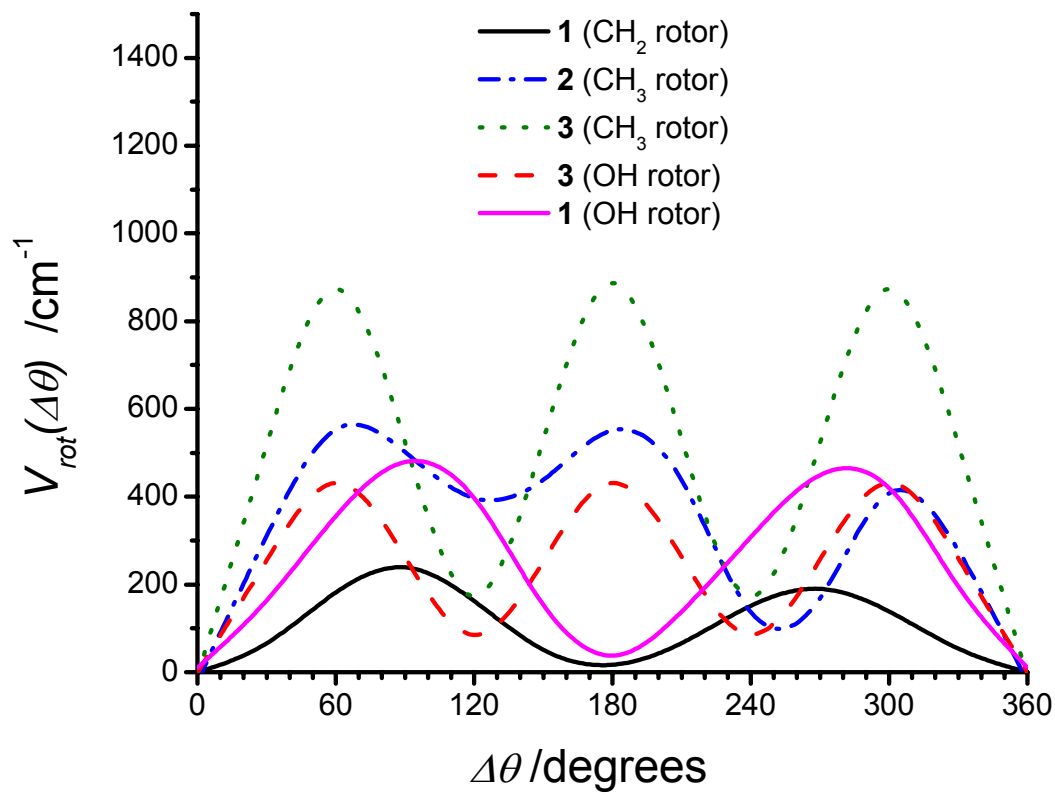

A. Rotational Potentials Calculated at the UB3LYP/6-311++G** Level 

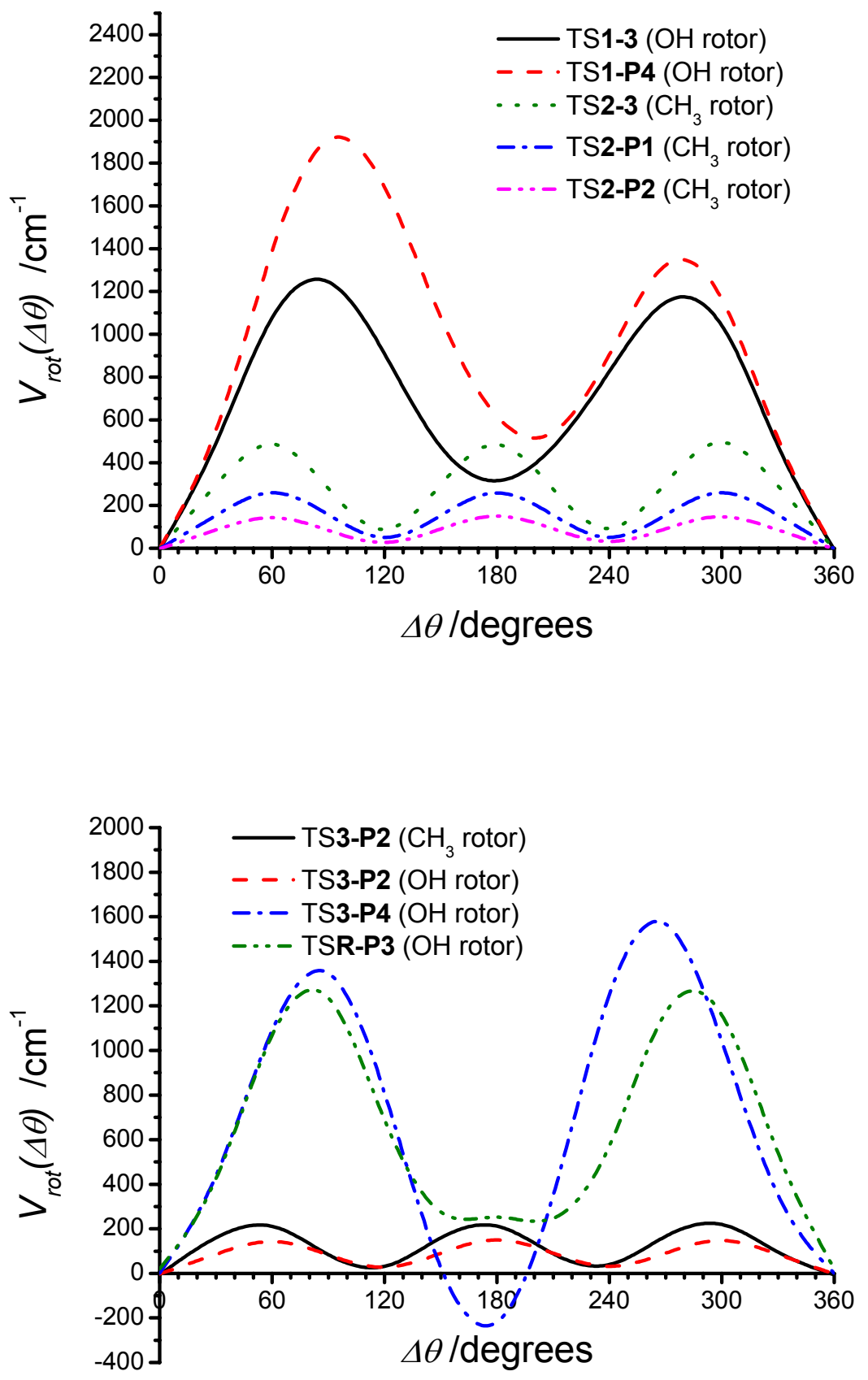


\title{
B. Variational Treatment of the Hydrogen Abstraction Channel
}

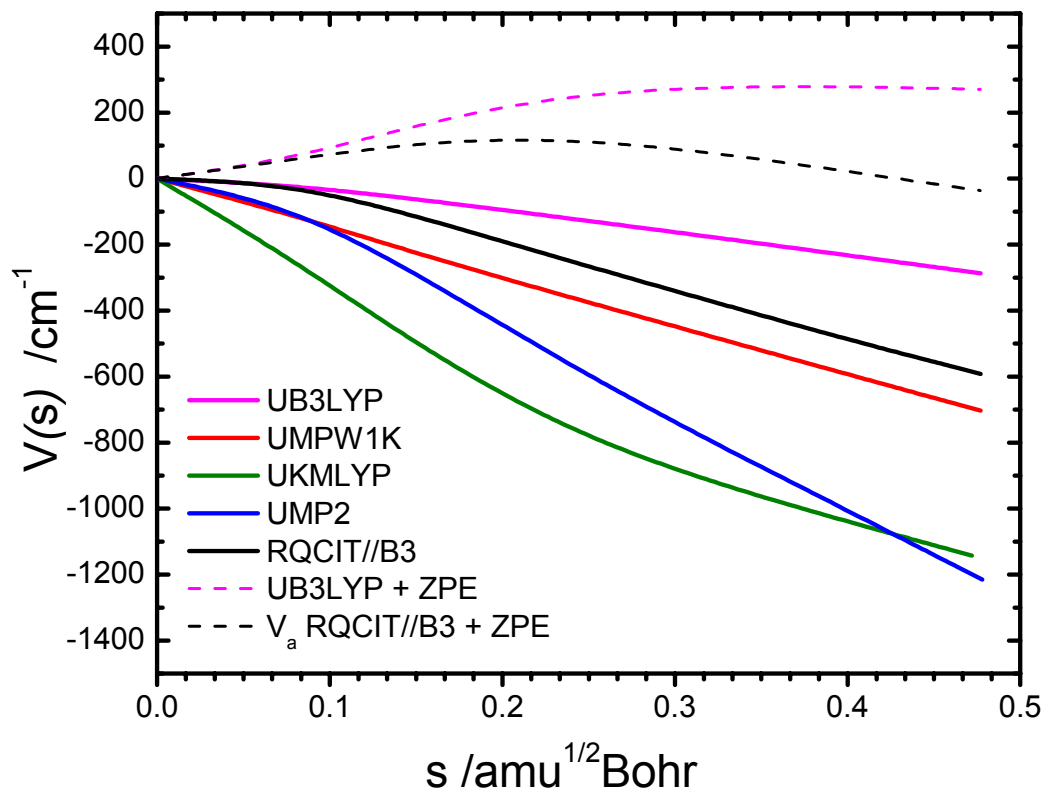

C. Cartesian Coordinates of Structures Optimized at the UQCISD/6-311++G(d,p) Level

\author{
C1 \\ C $-0.4160430 .670886-1.155694$ \\ C $-0.416057-0.670883-1.155698$ \\ H $0.5082581 .239942-1.228353$ \\ $\mathrm{H}-1.3410871 .239188-1.095442$ \\ H $0.508232-1.239958-1.228361$ \\ $\mathrm{H}-1.341113-1.239166-1.095450$ \\ O $0.783515-0.0000032 .156157$ \\ H $0.390191-0.0000021 .266703$
}

1

C 0.0000000 .0000000 .000000

C 0.0000000 .0000001 .495700

O $1.3173740 .000000-0.544325$

H -0.469325 $0.912932-0.394219$

H - $0.582976-0.852824-0.378803$

H 0.7244530 .6138312 .023978 
H $-0.831755-0.4237912 .051052$

H $1.781293-0.742848-0.151433$

$2\left(\mathrm{~A}^{\prime}\right)$

C 0.0000000 .0000000 .000000

C 0.0000000 .0000001 .544771

O $1.3271730 .000000-0.389688$

H - $-0.5176730 .902246-0.358018$

H - $0.517673-0.902246-0.358018$

H 0.5074110 .8912791 .924473

H $0.507411-0.8912791 .924473$

H -1.037173 0.0000001 .895602

$2(\mathrm{~A} ")$

C 0.0000000 .0000000 .000000

C 0.0000000 .0000001 .526000

O $1.2573570 .000000-0.573324$

$\mathrm{H}-0.5377020 .874884-0.409910$

H - $0.537702-0.874884-0.409910$

H 0.5160210 .8881631 .905360

H $0.516021-0.8881631 .905360$

H - 1.0268400 .0000001 .911447

3

C 0.0000000 .0000000 .000000

C 0.0000000 .0000001 .498769

O $1.2149900 .000000-0.648241$

$\mathrm{H}-0.7356580 .581156-0.548427$

H - $1.023793-0.0775241 .878330$

H $0.570902-0.8516511 .895009$

H 0.4496530 .9203271 .907967

H 1.836660 -0.502556 -0.115624

3 (trans)

C 0.0000000 .0000000 .000000

C 0.0000000 .0000001 .493600

O $1.2636460 .000000-0.551874$

H - $0.762912-0.552783-0.549367$ 
H - 1.0209240 .1182331 .871147 H $0.411570-0.9375341 .901355$ H 0.6124390 .8254171 .874731

H $1.177969-0.079043-1.503964$

$\operatorname{TS}(\mathbf{1} \leftrightarrow \mathbf{2})$

C 0.0000000 .0000000 .000000

C 0.0000000 .0000001 .504400

O 1.4487710 .0000000 .009184

H - $0.4004630 .901710-0.471941$

H - $0.399360-0.898835-0.471416$

H - 0.2398410 .9290372 .016919

H - $0.240120-0.9316062 .018285$

H 1.3256030 .0000001 .260135

$\operatorname{TS}(\mathbf{1} \leftrightarrow \mathbf{3})$

C 0.0000000 .0000000 .000000

C 0.0000000 .0000001 .482300

O $1.2521800 .000000-0.600213$

$\mathrm{H}-0.8071870 .529063-0.506813$

H - 0.7635170 .5491902 .022275

H - $0.423650-1.0005650 .727853$

H $0.923373-0.2805141 .975572$

H 1.161140 -0.404992 -1.465276

$\operatorname{TS}(\mathbf{1} \leftrightarrow \mathbf{P} 4)$

C 0.0000000 .0000000 .000000

C 0.0000000 .0000001 .362100

O $1.1185230 .000000-0.783958$

$\mathrm{H}-0.5881491 .749075-0.258270$

H - $0.876229-0.288117-0.571536$

H 0.9055620 .1887081 .933180

H - $0.942580-0.0516511 .894137$

H $1.8694150 .255434-0.241692$

$\operatorname{TS}(\mathbf{2} \leftrightarrow \mathbf{3})$

C 0.0000000 .0000000 .000000

C 0.0000000 .0000001 .503199 
O $1.2212390 .000000-0.661750$

H - $0.8182890 .508297-0.519233$

H 0.2850510 .9970671 .869329

H $-0.996361-0.2382281 .891004$

H $0.724645-0.7231341 .889549$

H $0.478873-0.960072-0.675294$

$\operatorname{TS}(\mathbf{2} \leftrightarrow \mathbf{P} \mathbf{1})$

C 0.0000000 .0000000 .000000

C 0.0000000 .0000002 .126800

O $1.2063350 .000000-0.281309$

H $-0.5894060 .933327-0.027544$

H $-0.589409-0.933325-0.027543$

H 0.5362050 .9235682 .322933

H - 1.0711550 .0001722 .317511

H $0.535904-0.9237482 .322908$

$\mathrm{TS}(\mathbf{2} \leftrightarrow \mathbf{P} 2)$

C 0.0000000 .0000000 .000000

C 0.0000000 .0000001 .518702

O $1.0362400 .000000-0.667923$

H - $0.9629960 .262822-0.482859$

H - $0.465313-1.664500-0.231990$

H 0.0569161 .0438041 .853808

H - $0.921072-0.4376981 .916617$

H $0.871374-0.5395071 .898292$

$\operatorname{TS}(\mathbf{3} \leftrightarrow \mathbf{P} 2)$

C 0.0000000 .0000000 .000000

C 0.0000000 .0000001 .502106

O $1.0527600 .000000-0.664644$

H - $0.9629510 .117274-0.522329$

H 0.1285831 .0388361 .844998

H $0.838125-0.5888881 .885183$

H - $0.945800-0.3787541 .901784$

H $1.9269241 .179827-0.532106$

$\mathrm{TS}(\mathbf{3} \leftrightarrow \mathbf{P 4})$ 
C 0.0000000 .0000000 .000000

C 0.0000000 .0000001 .351772

O $1.1029170 .000000-0.797813$

$\mathrm{H}-0.9089480 .081112-0.587273$

H $0.912045-0.1706861 .918297$

H -0.944136 -0.069031 1.878874

H 0.1786641 .9222951 .916309

H $1.883504-0.061585-0.239744$

\section{$\mathrm{TS}(\mathbf{R} \leftrightarrow \mathbf{1})$}

C 0.0000000 .0000000 .000000

C 0.0000000 .0000001 .362997

O 2.0939920 .0000001 .701704

H $0.117555-0.922735-0.561014$

$\mathrm{H}-0.0451400 .928282-0.564385$

$\mathrm{H}-0.050173-0.9302351 .918215$

H - 0.1874220 .9133861 .918155

H 2.3643070 .5458090 .947448

$\mathrm{TS}(\mathbf{R} \leftrightarrow \mathbf{P 3})$

C 0.0000000 .0000000 .000000

C 0.0000000 .0000001 .329561

H $0.9342450 .000000-0.561309$

$\mathrm{H}-0.925715-0.002930-0.570346$

H 0.8535270 .0018292 .002247

$\mathrm{H}-1.097276-0.0482841 .915813$

O -2.201661 0.1440292 .445421

H -2.167610 1.1101562.490476 